AperTO - Archivio Istituzionale Open Access dell'Università di Torino

\title{
Ion Beam Induced Charge analysis of diamond diodes
}

\section{This is the author's manuscript}

Original Citation:

Availability:

This version is available http://hdl.handle.net/2318/1660870

since 2018-02-27T13:40:42Z

Published version:

DOI:10.1016/j.nimb.2017.01.021

Terms of use:

Open Access

Anyone can freely access the full text of works made available as "Open Access". Works made available under a Creative Commons license can be used according to the terms and conditions of said license. Use of all other works requires consent of the right holder (author or publisher) if not exempted from copyright protection by the applicable law. 


\title{
Ion Beam Induced Charge analysis of diamond diodes
}

\author{
J. Lehnert ${ }^{1,2}$, J. Meijer ${ }^{1}$, C. Ronning ${ }^{3}$, D. Spemann ${ }^{1,2}$, E. Vittone ${ }^{4 *}$ \\ ${ }^{1}$ University Leipzig, Institute for Experimental Physics II, Department Nuclear Solid State Physics, Linnéstr. \\ 5, D-04103 Leipzig, Germany \\ ${ }^{2}$ Leibniz Institute of Surface Modification, Physical Department, Permoserstr. 15, D-04318 Leipzig, \\ Germany \\ ${ }^{3}$ Friedrich-Schiller-University of Jena, Institute for Solid State Physics, Max-Wien-Platz 1, D-07743 Jena, \\ Germany \\ ${ }^{4}$ University of Torino, Physics Department and NIS interdepartmental Centre, via P. Giuria 1, 10125 Torino, \\ Italy,
}

Keywords: Diamond diodes, Ion Beam Induced Charge (IBIC) technique, luminescent centres in diamond, electroluminescence.

\section{Abstract}

Diamond based p-i-n light-emitting diodes, developed to electrically drive single-photon sources in the visible spectral region at room temperature, have the potential to play a key role in quantum based technologies. In order to gain more insight into the charge injection mechanism occurring in these diodes, we carried out an experiment aimed to investigate the electrostatics and the charge carrier transport by the Ion Beam Induced Charge (IBIC) technique, using $1 \mathrm{MeV} \mathrm{He}$ microbeam raster scanning of $\mathrm{p}-\mathrm{i}-\mathrm{n}$ structures fabricated in a high purity diamond substrate, using lithographic masking and $\mathrm{P}$ and $\mathrm{B}$ ion implantation doping.

Charge Collection Efficiency (CCE) maps obtained at low ion fluence, show that induced charge pulses arise only from the P-implanted region, whereas no IBIC signals arise from the B-implanted region. This result suggests the formation of a slightly $\mathrm{p}$-type doped substrate, forming a $\mathrm{n}^{+}-\mathrm{p}-\mathrm{p}^{+}$, rather than the expected $\mathrm{p}-\mathrm{i}-\mathrm{n}$, structure.

However, for high fluence scans of small areas covering the intrinsic gap, CCE maps are more uniform and compatible with a p-i-n structure, suggesting the occurrence of a "priming effect", which saturates acceptor levels resulting in a decrease of the effective doping of the diamond substrate.

\footnotetext{
* Corresponding author: ettore.vittone@unito.it
} 


\section{Introduction}

One of the most interesting features of diamond diodes is electroluminescence induced by charge injection in recombination centers and, remarkably, in individual single nitrogen-vacancy (NV) centers located in the intrinsic region [1]. The possibility to develop diamond optoelectronic devices with stable, room-temperature, electrically driven single-photon sources is a key technology with a broad range of application ranging from quantum communication, computing and metrology [2]. The electrical control of the charge state of NV centers in diamond requires the control of the Fermi level in the diamond band-gap, which has been successfully achieved by incorporating the luminescent center in an intrinsic diamond layer sandwiched between graphitic/graphitic electrodes [3] or in p-i-n structures with graphitic ohmic contacts [4][5][6]. However, for the optimization of these devices and their standardization in the perspective of their large scale production, an accurate and spatially resolved characterization of their electrostatic features is essential.

This analysis can be effectively carried out by the Ion Beam Induced Charge (IBIC) technique, which has been widely proven to be suitable to provide valuable information on the electrostatic and transport characteristics of semiconductor/insulator electronic devices [7][8]. Besides, it is of high interest in the field of quantum technology, for example, for its potential to accurately measure the ion

strike location for single atom deterministic doping [9][10] in silicon and for the detection of single low energy ion $(\mathrm{Si}, 200 \mathrm{keV})$ in diamond for the optimization of the production yield of single colour centres [11].

However, to our best knowledge, IBIC technique has not been so far applied to the electronic characterization of $\mathrm{p}-\mathrm{i}-\mathrm{n}$ diamond structures, in order to extract electric field profiles, and carrier diffusion/drift lengths as done for example in $\mathrm{Si}, \mathrm{GaAs}, \mathrm{SiC}$ junction or Schottky diodes [7] [12].

In order to explore the potential of IBIC in this field, in this paper, we report on the first IBIC analysis of diamond-based p-i-n light-emitting diodes capable of single-photon emission in the visible spectral region at room temperature [4].

\section{Experimental}

The p-i-n diamond diodes were fabricated on an electronic grade ultra-pure highly polished (100)oriented single crystal CVD diamond film, $0.5 \mathrm{~mm}$ thick, using photolithographic masking and ion implantation doping. The p-type and n-type regions were realized by the implantation of $70 \mathrm{keV} \mathrm{B}$ ions with a fluence of $2 \cdot 10^{16}$ ions $/ \mathrm{cm}^{2}$ and $90 \mathrm{keV} \mathrm{P}$ ions with a fluence of $10^{16}$ ions $/ \mathrm{cm}^{2}$, respectively. 
Fig.1 summarizes the $\mathrm{P}$ and $\mathrm{B}$ implantation and the vacancy profiles evaluated by SRIM2013 simulations [13].

After B and P implantation, the sample was annealed at $1600^{\circ} \mathrm{C}$ for 4 hours in vacuum.

This annealing process had a double function: first to convert the highly damaged cap layers into graphite, with a vacancy density overcoming the graphitization threshold (assumed to be $\mathrm{V}_{\mathrm{G}}=10^{22}$ vacancies $\left./ \mathrm{cm}^{3}[14]\right)$. Second, to recover the diamond lattice from implantation damage and activate the $\mathrm{P}$ and $\mathrm{B}$ dopants located below this graphitized conductive layer, where the vacancy concentration is lower than $\mathrm{V}_{\mathrm{G}}$. The conductive graphitic cap layers act as ohmic contact to the buried doped regions and allow direct bonding to be performed without additional metallization [4].

The sample was finally mounted on a ceramic PCB and two diodes were connected to the PCB gold pads by $\mathrm{Al}$ wires $(20 \mu \mathrm{m})$ soldered on the top graphitic layers. The back side of the sample had no electrical contacts. The gaps (namely i-gaps) between the P- and B-doped regions of the two diodes under study, namely B3 and C4, were 7 and $9 \mu \mathrm{m}$, respectively. Figure 2 shows optical images of the sample and of one of the diodes with the electrical connections. The bias voltage was applied on the $\mathrm{n}^{+}$ electrode.

IBIC characterization was carried out at the LIPSION nanoprobe laboratory of the University of Leipzig [15], using $1 \mathrm{MeV}$ He ion beams (ion current $\approx 0.1 \mathrm{fA}$ ) focused down to $1 \mu \mathrm{m}$ spot size. The electronic energy loss profile, which corresponds to the electron/hole generation profile, induced by 1 $\mathrm{MeV}$ He ion in diamond extends to $1.8 \mu \mathrm{m}$ in depth.

The electronic chain was composed by an Amptek 250 charge sensitive pre-amplifier, a Canberra 2025 shaping amplifier (shaping time: $1 \mu \mathrm{s}$ ) and pulse heights were digitized by a Canberra 8701 ADC [16].

A Hamamatsu S1223-01 pin diode was used as the reference detector for charge collection efficiency (CCE) calibration (assuming 100\% CCE). The analysis of the $1 \mathrm{MeV}$ He IBIC spectrum provides a a spectral resolution of about $5 \mathrm{keV}$ in $\mathrm{Si}$; the noise threshold was set to channel 35 , corresponding to 6300 electrons ( $\approx 23 \mathrm{keV}$ in silicon). The CCE of diamond, was calculated assuming an electron/hole pair generation energy of $13 \mathrm{eV}$ [17].

IBIC maps (typically 256x256 pixels) were acquired by scanning the ion micro-beam over the sample surface, and recording the charge pulse heights as function of the beam position. The ADC and beam scanning were controlled by the MicroDAS data acquisition system [18] working in "clock triggering mode" with dwell times of $500 \mu \mathrm{s}$ and $1000 \mu \mathrm{s}$ in each pixel, depending on measurement. 
All the measurements were carried out in dark conditions [19] and at room temperature.

\section{Results and discussion}

Fig. 3 shows an IBIC map of a $720 \times 720 \mu \mathrm{m}^{2}$ scan including the whole p-n structure. The "shadows" of the bonding and Al wires make easy the correlation between this map and the diode photograph shown in Fig. 2b. It is apparent that only the phosphorous implanted region is visible, whereas the charge induced when $1 \mathrm{MeV}$ He ions probe the boron implanted region is null, or is below the electronic threshold (it should be noted that the B-doped region remained invisible in measurements with reversed polarity of the shaping amplifier). This result was unexpected, since the electric field in a $\mathrm{p}$-i-n diode arises from both the doped regions and extends deeply into the intrinsic (undoped) region.

However, if we assume that the bulk diamond is not perfectly intrinsic, but is slightly p-type doped, Fig. 3 is compatible with a single $n^{+}-p$ junction model, where the depletion region surrounds the $n^{+}-$ type (phosphorous doped) region and extends into the p-type region, whereas the $\mathrm{p}^{+}$-type (boron doped) region acts essentially as an injecting or ohmic contact to the p-type substrate.

In order to corroborate such an interpretation, we extracted CCE spectra from a selected region of the IBIC maps acquired at different bias voltage $\mathrm{V}_{\text {bias }}$ (Fig.4a). From the measurement of the CCE, it is possible to evaluate the depletion layer width (w), assuming a complete collection of induced charge generated in the depletion region and neglecting any diffusion effect, from the integral electron energy

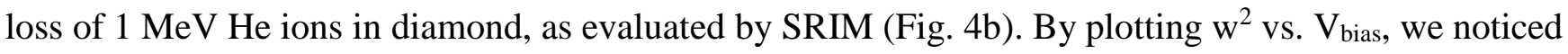
a linear behavior, which is compatible with the elemental Shockley model of an ideal $n^{+}-p$ junction diode in reverse bias [20];

$$
w^{2}=\frac{2 \cdot \varepsilon \cdot\left(V_{b i a s}+V_{b i}\right)}{q \cdot N_{A}}
$$

where $\varepsilon$ is the diamond dielectric constant $(\approx 0.5 \mathrm{pF} / \mathrm{cm})$ and $\mathrm{q}$ is the elementary charge.

From eq. (1), both the effective acceptor concentration $\mathrm{N}_{\mathrm{A}}=(1.50 \pm 0.08) \cdot 10^{16} \mathrm{~cm}^{-3}$ and the built-in potential of $\mathrm{V}_{\mathrm{bi}}=(3.7 \pm 0.6) \mathrm{V}$, can be extracted from the slope and the intercept of the linear fit (Fig. 4c).

It is worth noticing that the depletion region should extend not only in depth, i.e. underneath the $\mathrm{n}^{+}$ electrode, but also laterally, surrounding the whole $\mathrm{n}^{+}$electrode. However, the scarce resolution of the large-scan maps prevents a direct observation of the active region widening.

Although our elementary model provides values of built-in potential reasonably compatible with those relevant to diamond pn junctions available in literature [21], it is unsuitable to interpret IBIC 
maps generated by $1 \mathrm{MeV}$ He ion scans over smaller areas in between the two $\mathrm{P}$ and $\mathrm{B}$ implanted regions. Actually, both regions are visible in the $(22 \times 22) \mu \mathrm{m}^{2}$ CCE maps shown in Fig. 5, which have been acquired at different bias voltages. Furthermore, IBIC signals arise also from the i-gap with U- or $\mathrm{V}$ - shaped profiles at different voltages, which resemble the electric field profiles in an ideal p-i-n diode, which extends in the intrinsic (or slightly doped p-type) layer from the borders of both the $\mathrm{p}^{+}-$ and $\mathrm{n}^{+}$-type regions [22].

As the experimental conditions were identical to those adopted for the large scan map shown in Fig. 3 , the different response of the device has to be ascribed to the different ion fluences used for the two IBIC scans. In the former case $\left(720 \times 720 \mu \mathrm{m}^{2}\right.$ scan area), the fluence was of the order of $10^{7}$ ions $/ \mathrm{cm}^{2}$, whereas, for the detailed $\left(22 \times 22 \mu \mathrm{m}^{2}\right.$ scan area) map, the fluence was higher than $10^{10}$ ions $/ \mathrm{cm}^{2}$, corresponding to an absorbed dose within the irradiated volume, i.e. (irradiated area) $\mathrm{x}$ (ion range) $\approx$ $22 \times 22 \times 1.5 \mu^{3}$ ), of about $4 \mathrm{kGy}$. It has been extensively demonstrated that in mono- and polycrystalline diamond [23] [24], as well as in other wide band gap semiconductors [25], ionizing particle irradiation at high fluence induces a "priming" effect, which is usually explained by the saturation of active traps in the diamond bulk, which are filled by charge carriers generated by ionization. This priming effect suggests a tentative explanation of the controversial results given in Figs. 3 and 5: the high ionization rate induced by He ions during the small area scan passivate the effective acceptors in the diamond substrate responsible for the abovementioned $n^{+}-p$ junctions observed in large area IBIC maps (Fig. 3). Therefore, the region irradiated at high fluence assumes an almost intrinsic nature, which is compatible with the formation of a depletion region extending over the entire irradiated area. The electric field is then supposed to permeate the whole i-gap and the surroundings of the $\mathrm{p}^{+}$and $\mathrm{n}^{+}$ regions up to the ionization depth of the $1 \mathrm{MeV} \mathrm{He}$ ions (around $1.5 \mu \mathrm{m}$ ), promoting the drift of carriers generated by ionization, and hence the induction of charge at the sensing electrode.

\section{Conclusions}

In this work, we have for the first time performed an IBIC analysis of p-i-n diamond structures. Two significant and unexpected results have been achieved:

1) Large area/low fluence IBIC maps show only the phosphorous implanted region, whereas both the i-gap and the boron implanted regions are inactive for charge induction. The confinement of the electric field at the $\mathrm{n}^{+}$type region contradicts the hypothesis of an intrinsic diamond substrate, whereas is compatible with the presence of a unique $n^{+}-p$ junction. Therefore, it seems that the high temperature annealing plays a role not only for the electrical activation of dopants 
in the $\mathrm{P}$ and $\mathrm{B}$ implanted regions, but also to activate acceptor impurity/defects in the diamond substrate, with an effective concentration of $1.5 \cdot 10^{16} \mathrm{~cm}^{-3}$.

2) Both the P- and B-implanted regions and the i-gap are IBIC active only if they are irradiated with high ion fluence. This effect has been interpreted as due to the saturation of the acceptor levels in the bulk induced by ionization, resulting in a sort of dopant compensation, which drastically reduces the effective p-type doping observed during low fluence scans. Under these high fluence conditions, IBIC maps show features typical of p-i-n structures.

It is worth underlying that our model is rather elementary and does take into account neither the complex structure of the device shown in Fig. 1, which generates a complex electric field profile due to the combination of rectifying, ohmic (graphitic), injecting contacts, nor the possible induction of "anomalous polarity pulses" due to a selective recombination of carriers before collection at the

electrodes [26]. More experiments and more computational efforts will be needed to definitely validate our interpretation

However, this phenomenological model seems to be sufficient for a basic interpretation of the experimental findings and, in general, it can also provide some remarks regarding the IBIC technique which deserve some consideration: point 1) once more highlights the sensitivity of the IBIC technique for the functional characterization of semiconductor/insulator devices, revealing details not observable with other standard techniques.

On the other hand, point 2) evidences that the IBIC technique cannot always be considered a fully non-invasive technique: as apparent in highly resistive materials subjected to "priming effects", where the high charge density induced by high ion fluence can remarkably perturb the electronic features of the device under study.

\section{Acknowledgements}

This work has been carried out within the IAEA coordinated research project F11016. "Utilization of Ion Accelerators for Studying and Modelling of Radiation Induced Defects in Semiconductors and Insulators". JL, JM and DS are financial supported by the VolkswagenStiftung, DFG FOR 1493 and the EU DIADEMS Project.

\section{References}


[1] N. Mizuochi, T. Makino, H. Kato, D. Takeuchi, M. Ogura, H. Okushi, et al., Electrically driven single-photon source at room temperature in diamond, Nat. Photonics. 6 (2012) 299-303. doi:10.1038/nphoton.2012.75.

[2] I. Aharonovich, E. Neu, Diamond nanophotonics, Adv. Opt. Mater. 2 (2014) 911-928. doi:10.1002/adom.201400189.

[3] J. Forneris, P. Traina, D.G. Monticone, G. Amato, L. Boarino, G. Brida, et al., Electrical stimulation of non- classical photon emission from diamond color centers by means of sub-superficial graphitic electrodes, Sci. Rep. (2015) 1-7. doi:10.1038/srep15901.

[4] A. Lohrmann, S. Pezzagna, I. Dobrinets, P. Spinicelli, V. Jacques, J.F. Roch, et al., Diamond based light-emitting diode for visible single-photon emission at room temperature, Appl. Phys. Lett. 99 (2011). doi:10.1063/1.3670332.

[5] M. Shimizu, T. Makino, T. Iwasaki, J. Hasegawa, K. Tahara, W. Naruki, et al., Charge state modulation of nitrogen vacancy centers in diamond by applying a forward voltage across a p-i-n junction, Diam. Relat. Mater. 63 (2016) 192-196. doi:10.1016/j.diamond.2015.10.022.

[6] S. Pezzagna, J. Meijer, Single-Ion Implantation in Diamond with a High Lateral Resolution: A Key Technology for the Fabrication of Quantum Devices, in: Compr. Hard Mater., Elsevier Ltd., 2014: pp. 321-336. doi:10.1016/B978-0-08-096527-7.00050-7.

[7] M.B.H. Breese, E. Vittone, G. Vizkelethy, P.J. Sellin, A review of ion beam induced charge microscopy, Nucl. Instruments Methods Phys. Res. Sect. B Beam Interact. with Mater. Atoms. 264 (2007) 345-360. doi:10.1016/j.nimb.2007.09.031.

[8] E. Vittone, Z. Pastuovic, M.B.H. Breese, J. Garcia Lopez, M. Jaksic, J. Raisanen, R. Siegele, A. Simon, G. Vizkelethy, Charge collection efficiency degradation induced by $\mathrm{MeV}$ ions in semiconductor devices: Model and experiment, Nucl. Instruments Methods Phys. Res. Sect. B Beam Interact. with Mater. Atoms. 372 (2016) 128-142. doi:10.1016/j.nimb.2016.01.030.

[9] D.N. Jamieson, C. Yang, T. Hopf, S.M. Hearne, C.I. Pakes, S. Prawer, et al., Controlled shallow single-ion implantation in silicon using an active substrate for sub- 20-keV ions, Appl. Phys. Lett. 86 (2005) 1-3. doi:10.1063/1.1925320.

[10] J. Forneris, D.N. Jamieson, G. Giacomini, C. Yang, E. Vittone, Modeling of ion beam induced charge sharing experiments for the design of high resolution position sensitive 
detectors, Nucl. Instruments Methods Phys. Res. Sect. B Beam Interact. with Mater. Atoms. 306 (2013) 169-175. doi:10.1016/j.nimb.2012.12.025.

[11] J.B.S. Abraham, B.A. Aguirre, J.L. Pacheco, G. Vizkelethy, E. Bielejec, Fabrication and characterization of a co-planar detector in diamond for low energy single ion implantation, Appl. Phys. Lett. 109 (2016) 63502. doi:10.1063/1.4960968.

[12] E. Vittone, Z. Pastuovic, P. Olivero, C. Manfredotti, M. Jaksic, a. Lo Giudice, et al., Semiconductor characterization by scanning ion beam induced charge (IBIC) microscopy, Nucl. Instruments Methods Phys. Res. Sect. B Beam Interact. with Mater. Atoms. 266 (2008) 1312-1318. doi:10.1016/j.nimb.2007.12.083.

[13] J.F. Ziegler, M.D. Ziegler, J.P. Biersack, SRIM - The stopping and range of ions in matter (2010), Nucl. Instruments Methods Phys. Res. Sect. B Beam Interact. with Mater. Atoms. 268 (2010) 1818-1823. doi:10.1016/j.nimb.2010.02.091.

[14] C, Uzan-Saguy, C. Cytermann, R. Brener, V. Richter, M. Shaanan, R. Kalish, (1995) 'Damage threshold for ion-beam-induced graphitization of diamond', Applied Physics Letters, 67(9), pp. 1194-1196. doi: 10.1063/1.115004.

[15] D. Spemann, T. Reinert, J. Vogt, T. Andrea, N. Barapatre, R. Feder, et al., Materials analysis and modification at LIPSION - Present state and future developments, Nucl. Instruments Methods Phys. Res. Sect. B Beam Interact. with Mater. Atoms. 269 (2011) 2175-2179. doi:10.1016/j.nimb.2011.02.054.

[16] A.M. Jakob, D. Spemann, R. Thies, J. Barzola-Quiquia, J. Vogt, T. Butz, A characterisation of electronic properties of alkaline texturized polycrystalline silicon solar cells using IBIC, Nucl. Instruments Methods Phys. Res. Sect. B Beam Interact. with Mater. Atoms. 269 (2011) 2345-2349. doi:10.1016/j.nimb.2011.02.040.

[17] J. Kaneko, M. Katagiri, Diamond radiation detector using a synthetic IIa type monocrystal, Nucl. Instruments Methods Phys. Res. Sect. A Accel. Spectrometers, Detect. Assoc. Equip. 383 (1996) 547-548. doi:10.1016/S0168-9002(96)00868-6.

[18] A. Sakellariou, G.R. Moloney, D.N. Jamieson, Dead time corrected and charge normalised maps generated with the MicroDas fast data acquisition system, Nucl. Instruments Methods Phys. Res. Sect. B Beam Interact. with Mater. Atoms. 181 (2001) 116-121. doi:10.1016/S0168-583X(01)00617-6. 
[19] J.A. Dueñas, J. de la Torre Pérez, A. Martín Sánchez, I. Martel, Diamond detector for alpha-particle spectrometry, Appl. Radiat. Isot. 90 (2014) 177-180. doi:10.1016/j.apradiso.2014.03.032.

[20] E. Vittone, Theory of ion beam induced charge measurement in semiconductor devices based on the Gunn's theorem, Nucl. Instruments Methods Phys. Res. Sect. B Beam Interact. with Mater. Atoms. 219-220 (2004) 1043-1050. doi:10.1016/j.nimb.2004.01.210.

[21] T. Makino, H. Kato, S.G. Ri, S. Yamasaki, H. Okushi, Homoepitaxial diamond p-n+ junction with low specific on-resistance and ideal built-in potential, Diam. Relat. Mater. 17 (2008) 782-785. doi:10.1016/j.diamond.2007.12.006.

[22] S.M. Sze, K.K. Ng, Physics of Semiconductor Devices, J. Wiley, NJ 2007. doi:10.1049/ep.1970.0039.

[23] C. Manfredotti, F. Fizzotti, L. Giudice, C. Paolini, E. Vittone, R. Lu, Homogeneity of CVD diamond detectors in tracking applications, Nucl. Instruments Methods Phys. Res. Sect. B Beam Interact. with Mater. Atoms. 187 (2002) 566-578. doi:10.1016/S0168$583 X(01) 01160-0$.

[24] A. Lohstroh, P.J. Sellin, S. Gkoumas, J. Parkin, P. Veeramani, G. Prekas, et al., Ion beam induced charge (IBIC) irradiation damage study in synthetic single crystal diamond using 2.6 MeV protons, Phys. Status Solidi Appl. Mater. Sci. 205 (2008) 2211-2215. doi:10.1002/pssa.200879701..

[25] A. Lohstroh, P. Sellin, a Simon, High-resolution mapping of the mobility-lifetime product in CdZnTe using a nuclear microprobe, 16 (2004) 67-73. doi:10.1088/0953$8984 / 16 / 2 / 008$.

[26] J. Forneris, V. Grilj, M. Jakšić, P. Olivero, F. Picollo, N. Skukan, et al., Measurement and modelling of anomalous polarity pulses in a multi-electrode diamond detector, EPL (Europhysics Lett. 104 (2013) 28005. doi:10.1209/0295-5075/104/28005. 


\section{Figure Captions:}

Fig. 1: a) Vacancy and implanted $B$ profiles $\left(70 \mathrm{keV}\right.$, fluence $\left.=2 \cdot 10^{16} \mathrm{~cm}^{-2}\right)$; b) vacancy and implanted $P$ profiles $\left(90 \mathrm{keV}\right.$, fluence $\left.=10^{16} \mathrm{~cm}^{-2}\right)$. The horizontal line indicates the graphitization threshold $\mathrm{V}_{\mathrm{G}}$. Regions on the left side of the vertical lines are relevant to highly damaged layers, with vacancy densities higher than $V_{G}$, which convert to graphite following the annealing process. On the right side of the vertical lines, the vacancy density is lower than $V_{G}$ and the annealing process promotes both the recovery of the diamond lattice and the activation of dopants, providing $\mathrm{p}$ - and n-type buried layers (bright red and green regions for $\mathrm{B}$ and $\mathrm{P}$ doping, respectively).

Fig. 2: a) Optical images of the diamond sample with the p-n junction diodes, c) optical image in transmittance of one diode with the scheme of the electronic connections.

Fig. 3: IBIC map of a p-n structure similar to the one shown in Fig. 2c. Reverse bias voltage (10 V).

Fig. 4: a) IBIC spectra from a selected region of the n-type region at different bias voltage; b) Normalized Ionization Energy Loss evaluated from Fig. 1b as function of depth; vertical colored segments indicate the depletion layer widths in correspondence of the median CCE values of the spectra at different bias voltages (colored horizontal lines); c) square of the depletion layer width as function of the bias voltage

Fig. 5: (top) CCE maps of a $(22 \times 22) \mu \mathrm{m} 2$ region covering the $\mathrm{n}$ - and $\mathrm{p}$ - regions at different bias voltage. (bottom) CCE profiles extracted from the regions limited by white lines. Red lines border the i-gap region in between the $\mathrm{n}$ - and $\mathrm{p}$ - regions. 